\author{
Aleksandra Burdziej \\ ORCID: 0000-0002-8567-2177 \\ Nikolaus-Kopernikus-Universität Toruń
}

DOI: $10.19195 / 0435-5865.144 .8$

\title{
Zwischen Unterhaltung und „Wissenschaft“" Zum Katastrophennarrativ in Marc Elsbergs Roman Blackout
}

\begin{abstract}
s
Ziel des vorliegenden Aufsatzes ist es, den Roman Blackout von Marc Elsberg vor dem Hintergrund der gegenwärtigen, von der amerikanischen Massenkultur stark geprägten Katastrophennarrationen zu diskutieren. Im Gegensatz zu vielen Katastrophengeschichten unserer Zeit, die wenig plausible, unrealistische oder gar absurde Bedrohungsszenarien zeichnen, entwirft Elsberg das absolut realistische Szenario eines großflächigen und langfristigen Stromausfalls und seiner Folgen für die Menschheit. Zwar spielt seine Prosa durchaus mit zeittypischen gesellschaftlichen Ängsten, gleichwohl soll sie, so zumindest die deklarierte Absicht des Autors, auch für einen bewussten Umgang mit neuen Technologien sensibilisieren. Nicht von ungefähr stützen sich seine Texte auf intensive wissenschaftliche Recherche. Vor dieser Folie lässt sich Elsbergs Prosa auch als eine Art „Warnungsliteratur" lesen und deuten.
\end{abstract}

Schlüsselwörter: Blackout, Stromausfall, Cyberterrorismus, Katastrophe, Katastrophennarrativ, "Warnungsliteratur"

\section{Between entertainment and "science": On catastrophic narrative in Marc Elsberg's novel Blackout}

The aim of this paper is to discuss Marc Elsberg's novel Blackout against the background of contemporary catastrophic narratives, strongly shaped by American mass culture. Contrary to numerous stories of catastrophes and dystopian visions of our time that tend to present unconvincing, unrealistic or even absurd threats, Elsberg presents a completely realistic scenario of a long term and extensive blackout, as well as its consequences for humanity. While his prose builds on contemporary fears, at least in the author's declaration, however, it seems to aim at increasing social awareness of the conscious use of new technologies. His book is based on intense desk research and incorpor- 
ates scientific evidence. Against this background, Elsberg's novel could be read and interpreted as "literature of warning".

Keywords: Blackout, cyberterrorism, catastrophe, catastrophic narrative, "literature of warning"

Aleksandra Burdziej, Uniwersytet Mikołaja Kopernika w Toruniu, Katedra Filologii Germańskiej, ul. Bojarskiego 1, 87-100 Toruń, Polen, E-Mail: aburdziej@umk.pl

Received: 30.09.2018, accepted: 8.04.2019

\section{Einleitung}

Katastrophen haben die Menschen von Anfang an begleitet, so dass sie die Kunst seit jeher inspirierten, darunter auch die Literatur. In der gegenwärtigen, immer mehr von (globalen) Krisen und Risiken geprägten Welt scheinen Katastrophengeschichten sowie dystopische Visionen unterschiedlicher Provenienz wieder deutlich an Bedeutung zu gewinnen (vgl. Walter 2010). Die Zahl und die Vielfalt von Katastrophenromanen, Katastrophenfilmen oder -serien der letzten Zeit - sei es auch der letzten Dekade - ist überraschend und markant. ${ }^{1}$ Darunter ist eine große Anzahl von Narrationen zu verzeichnen, in denen - meistens hyperbolisch - globale Naturkatastrophen gezeigt werden. ${ }^{2}$ Parallel dazu lässt sich ein merkwürdiger Mangel an utopischen Visionen oder zumindest an einigermaßen optimistischen Zukunftsentwürfen feststellen. Zum Teil resultiere dieses Phänomen aus einem erzählerischen Problem: Karlheinz Steinmüller verweist hier auf die Tatsache, dass die Darstellung einer idealen, harmonischen Gesellschaft in der Regel „keine sinnvollen handlungstragenden Konflikte“ biete (Steinmüller 1995: 88). Diese Tatsache könnte aber auch von einem vorwiegend zukunftspessimistischen Zeitgeist zeugen. Edwin Bendyk schreibt, dass der „hochmütige Glaube“ an die Kontrolle über die Zukunft (dass sich die Zukunft sowohl erraten, als auch aktiv gestalten lässt) definitiv verschwunden sei, als 1980 im französischen Magazin „Le debat“ ein bahnbrechender Essay von Krzysztof Pomian veröffentlicht wurde. Der bekannte polnische Philosoph habe in seinem Text überzeugend gezeigt, dass das menschliche „Instrumentarium“, das bisher unser Zukunftsdenken beherrscht habe, seine „Macht“ bereits „verloren“ habe. Denn:

${ }^{1}$ Siehe z.B. den Text von Marcin Zwierzchowski unter dem vielsagenden Titel: „Wir träumen nur noch Alpträume“ [Übersetzung des Titels - A. Burdziej]. (Zwierzchowski 2016)

${ }^{2}$ Siehe z.B. nur den Filmbereich; da sind in den letzten etwa zehn Jahren u.a. zu verzeichnen: San Andreas (2015), Regie: Brad Peyton; Storm Hunters (2014), Regie: Steven Quale; Noah (2014), Regie: Darren Aronofsky; Pompeii (2014), Regie: Paul W.S. Anderson; The Impossible (2012), Regie: Juan Antonio Bayona; Hell (2011), Regie: Tim Fehlbaum; 2012 (2009), Regie: Roland Emmerich; Sunshine (2007), Regie: Danny Boyle; The Day After Tomorrow (2004), Regie: Roland Emmerich; Volcano (1997), Regie: Mick Jackson. 
Wir können nicht mehr so auf den Glauben vertrauen, wie es vormoderne Menschen getan haben, aber wir haben auch unser Vertrauen zur Wissenschaft verloren, als wir uns überzeugt haben, dass die Folgen des wissenschaftlich-technischen Fortschrittes gegen den Menschen gerichtet werden können. In dieser Situation haben auch die Literatur und - breiter - Kunst und Kultur angefangen, engagiert das Thema Apokalypse als die wahrscheinlichste Vision der Zukunft aufzugreifen. (Zit. nach Bendyk 2016: 15) ${ }^{3}$

Auf den ersten Blick scheint der Roman Blackout des österreichischen Schriftstellers Marc Elsberg diesem Trend zu folgen. Der Roman erzählt nämlich von den katastrophalen Auswirkungen eines unerwarteten, großflächigen Stromausfalls in Europa und in den USA. In den fünfzehn Kapiteln, die jene Tage ohne Strom darstellen, schaut der Leser zu, wie plötzlich auf stockdunklen Kreuzungen Autos ineinander rasen, wie Menschen in U-Bahnen eingesperrt werden, Fahrstühle zwischen zwei Stockwerken hängen bleiben, U-Bahnen feststecken, Kraftwerke sich abschalten. In den Zentralen der Stromversorger und Netzbetreiber herrscht zugleich höchste Alarmstufe. Denn einmal aus dem Gleichgewicht gebracht, sind die fragilen Energienetze kaum noch zu stabilisieren - die Kettenreaktion ist nicht mehr aufzuhalten. Entsetzt müssen im Roman die Experten mit ansehen, wie in ganz Europa die Stromversorgung zusammenbricht. Es stellt sich bald heraus, dass hinter dem Blackout ein gezielter Cyberangriff steckt. Manipuliert wurden die intelligenten Stromzähler, die in allen Haushalten in Italien und Schweden installiert worden sind. Die Situation wird in „Blackout“ aber bald noch dramatischer: Auch in den USA fallen die Netze aus. Durch Kälte, Nahrungsmangel, fehlende medizinische Versorgung und heftige Unruhen sterben tausende Menschen. Als mehrere europäische Atomkraftwerke vor der Havarie stehen und Millionen Menschen bedrohen, beginnt ein Wettlauf mit der Zeit. Denn die Welt steht vor dem Abgrund. ${ }^{4}$ All dies wird in einem als dokumentarisch zu bezeichnenden, aber doch sehr bildhaften Stil dargestellt. Dies wird u.a. an jenen Stellen deutlich, an denen sehr realistisch anmutende, weil alarmierende Presseberichte aus aller Welt in den Text eingeflochten und von Protagonisten kommentiert werden. Der Realitätsbezug wird durch eine Vielzahl an eingeführten Protagonisten aus aller Welt unterstrichen, deren Beziehungen zueinander Schritt für Schritt enthüllt werden. Diese Vielstimmigkeit und Multiperspektivität, die stellenweise so authentisch und überzeugend wirken, werden im ganzen Text beibehalten.

Das Hauptthema des Romans von Elsberg ist also unbestritten eine Katastrophe, von erschreckendem, weil fast globalem Umfang. Von den heutigen Katastrophennarrationen und dem modischen Katastrophismus unterscheidet sich Blackout von Marc Elsberg dennoch. Der Unterschied wird klar, wenn man die im Roman präzise geschilderten Umstände der Katastrophe, ihre Ursachen und Folgen sowie des Weiteren auch die Art und Weise, wie sie bewältigt wird, ein-

${ }^{3}$ Vgl. auch: Zwierzchowski (2016: 100-104). Übersetzung aus dem Polnischen: A. Burdziej.

${ }^{4}$ Fragmente der Zusammenfassung von Blackout direkt oder etwas umformuliert übernommen aus: Sembritzki (2012). 
gehender analysiert. Zunächst aber wird im Weiteren das Konzept zur amerikanischen Massenkultur von Robert Jewett und John Shelton Lawrence beschrieben, das ich als theoretischen und durchaus aufschlussreichen Rahmen für die Analyse und Interpretation des Romans von Elsberg betrachte.

\section{Katastrophenszenarien der heutigen Massenkultur und das Konzept von Robert Jewett und John Shelton Lawrence}

Katastrophenszenarien in Filmproduktionen und Texten der gegenwärtigen Massenkultur wirken meist unrealistisch und wenig plausibel. Neben Naturkatastrophen, die z.B. eine neue Eiszeit, eine andauernde Hitze oder katastrophale Erdbeben schildern, ${ }^{5}$ und die theoretisch mögliche Naturerscheinungen (z.B. als Folge des Klimawandels) übertrieben zeigen und somit eine Art Hyperbel darstellen, werden auch Katastrophen weltweiten Ausmaßes dargestellt, die von übernatürlichen Kräften, gruseligen Fremdwesen oder von angreifenden Außerirdischen verursacht worden sind. Letzteres wirkt als Ursache von globalen Katastrophen häufig besonders unrealistisch und nicht selten karikaturistisch oder absurd. Als Weltretter findet sich da am Ende oft ein „Superheld“, der über übernatürliche Kräfte oder eine spezielle, für den üblichen Sterblichen unzugängliche Ausrüstung verfügt, und dem die erschöpfte, dezimierte Menschheit ihr weiteres Leben zu verdanken hat. ${ }^{6}$

Anders ist dies bei Elsberg. Einen erhellenden Rahmen für die Deutung von Blackout bietet das Konzept von Robert Jewett und John Shelton Lawrence, das sie in ihrem Buch Captain America and the Crusade Against Evil: The Dilemma of Zealous Nationalism entwickelt haben (Jewett/Lawrence 2004). ${ }^{7}$ Die Thesen von Jewett und Lawrence dienen der Erklärung des Einflusses, den die Massenkultur auf die Entwicklung der amerikanischen Zivilreligion (civil religion) genommen hat und vor allem des Phänomens der Popularität von amerikanischen Superhelden. Aber auch bei der Interpretation von Blackout kann die erwähnte Theorie Anwendung finden, da sie einen durchaus interessanten Hintergrund liefert.

${ }^{5}$ Vgl. Fußnote 3.

${ }^{6}$ Ausgewählte Superheldenfilme (2011-2016): Captain America: Civil War (2016), Regie: Anthony Russo, Joe Russo; X-Men: Apocalypse (2016), Regie: Bryan Singer; Batman v Superman: Dawn of Justice (2016), Regie: Zack Snyder; Avengers: Age of Ultron (2015), Regie: Joss Whedon; Teenage Mutant Ninja Turtles (2014), Regie: Jonathan Liebesman; X-Men: Zukunft ist Vergangenheit (2014), Regie: Bryan Singer; The Amazing Spider-Man 2: Rise of Electro (2014), Regie: Marc Webb; The Return of the First Avenger (2014), Regie: Anthony Russo, Joe Russo; Man of Steel (2013), Regie: Zack Snyder; Iron Man 3 (2013), Regie: Shane Black; The Amazing Spider-Man (2012), Regie: Marc Webb; Captain America: The First Avenger (2011), Regie: Joe Johnston.

${ }^{7}$ Vgl. auch ihre früheren Arbeiten: Jewett/Lawrence (1997); Lawrence/Jewett (2002). 
In ihren Ausführungen befassen sich Jewett und Lawrence unter anderem mit der Verbreitung und Beliebtheit, derer sich Superhelden, aber auch die Superheldenproduktion in den USA seit Jahrzehnten erfreut. ${ }^{8}$ Als Hauptheld fungiert in solchen Kunstwerken und ,Genres' (seien es Texte, Comics oder Filmproduktionen) immer ein Held, der entweder über Superkräfte oder spezielle Superfähigkeiten verfügt, oder dessen Überlegenheit auf enormer Intelligenz, Willenskraft sowie hartem Training basiert. Er taucht in der Regel auf, wenn die Menschen nicht mehr fähig sind, eine Gefahr selbstständig zu bewältigen und sich die Situation auf dramatische Weise zuspitzt. Im allerletzten Moment aber rettet er die Menschheit, die Welt oder eine bestimmte Gemeinschaft doch noch. Superman, Batman, Spider-Man, Iron Man - alle diese heroes sind Erzeugnisse der amerikanischen Massenkultur, deren Ursprung in einer amerikanischen Comicfigur, nämlich in Captain America, zu finden ist. Dieser Superheld, der in den Farben der Flagge der Vereinigten Staaten erscheint, wurde 1941, d.h. in Kriegszeiten, geschaffen. Ein unscheinbarer, schwächlicher Steve Rogers, den man als ungeeignet für den Wehrdienst betrachtet, wird dank des Experiments eines verrückten Wissenschaftlers zu einem muskulösen Supersoldaten; gleichzeitig werden ihm bestimmte Superkräfte verliehen. Vereinsamt und anonym kämpft er daraufhin gegen das nationalsozialistische Deutschland - denn offiziell ist er immer noch ein gewöhnlicher Militärbeamter. Dieses Muster, das zwecks Kriegspropaganda ins Leben gerufen wurde, wurde in den USA in unzählbaren Varianten von der Massenkultur aufgegriffen - in Filmen, Comics, Büchern und in Computerspielen, die vor allem an die Jugend adressiert wurden. Superman, Spider-Man, Batman, Iron Man usw. wiederholen stets aufs Neue das Schema des Einzelgängers - eines Außenseiters - der, der jedes Mal einer übertrieben dargestellten Gefahr allein die Stirn bietet. Dabei rettet er die hilflose Menschheit in den meisten Fällen mithilfe rücksichtsloser Gewalt (vgl. Burdziej 2005).

Laut Jewett und Lawrence ist hier besonders die Tatsache auffällig, dass sich die demokratischen, staatlichen oder lokalen Institutionen stets als ineffektiv erweisen, um die Katastrophe zu verhindern. Es müsse immer zur Intervention eines Superhelden kommen, denn nur er sei fähig, das Böse zu bewältigen. Und der Kampf mit

8 Jewett und Lawrence entwickeln in ihrem Buch (Jewett/Lawrence 2004) ein einheitliches Konzept des geistigen Zustands der Amerikaner, in dem sie von zwei Strömungen der amerikanischen Zivilreligion ausgehen - den ,prophetischen Realismus“ (profetic realism) sowie den „eifrigen Nationalismus“" (zealous nationalism). Die beiden sind tief in der biblischen Tradition verwurzelt und als zwei gegensätzliche Haltungen der gesellschaftlich-politischen Wirklichkeit gegenüber kämpfen sie laut der Autoren bis heute um Vorrang in der Gestaltung der Außenpolitik der Vereinigten Staaten. Sie beeinflussen auch stark die gegenwärtige Massenkultur (vgl. auch Burdziej 2005). In meinem Aufsatz konzentriere ich mich nur auf einen der mehreren Kontexte der Ausführungen von Jewett und Lawrence, den ich besonders interessant finde und der - meiner Meinung nach - den Roman von Elsberg in einem neuen Licht erscheinen lässt - auf den Kontext der gegenwärtig von der amerikanischen Massenkultur propagierten Bilder und Verhaltensmuster sowie des Verhältnisses zur Demokratie. 
dem Bösen rechtfertigt die grausamste Gewalt. Jewett und Lawrence analysieren die Botschaft, die diese Produkte der amerikanischen Massenkultur mit sich tragen und richten gegen solche Szenarien schwere Vorwürfe - vor allem deswegen, weil sie oft von jungen Menschen wahrgenommen werden und den nächsten Generationen verfälschte Handlungsmuster und Haltungen einprägen. Die Autoren behaupten, dass solche Superheldengeschichten und die Art und Weise, wie in ihnen die Katastrophen bewältigt werden, den Glauben an Wirksamkeit und Effektivität der Demokratie untergraben würden. Angesichts der Bedrohung seien die demokratisch gewählten Regierungen, der Staat sowie seine Institutionen und Behörden angeblich nicht imstande, die Bevölkerung zu verteidigen. Um der Katastrophe ein Ende zu setzen, müsse ein Superheld kommen. Er sei es, der die Macht habe, die dargestellte Realität zu ändern. Im Kampf mit der Bedrohung werde dabei aber jegliche Art der Gewalt gerechtfertigt. Die Bewältigung der Katastrophe verdanke sich daher nicht der gemeinsamen Anstrengung und Mühe vieler Menschen, die zusammen im Rahmen demokratischer Strukturen zusammenarbeiten und sich engagieren, sondern nur einer einzigen, quasi übermenschlichen Figur. Und der Superheld handele nicht auf dem Wege des Dialogs oder der Verhandlung, sondern der eigenen, autark gesetzten Moral und Gewalt entsprechend. Die letztere scheine übrigens in der schwarz-weißen Welt - und wenn die demokratischen Strukturen scheitern - der einzige sinnvolle Weg zu sein, laute die von solchen Narrationen vermittelte Botschaft, so Jewett und Lawrence (Jewett/Lawrence 2004: 26-43). ${ }^{9}$

Die amerikanische Massenkultur ist im Zeitalter der Globalisierung zwangsläufig weltweit präsent. Besonders stark ist sie nach Europa durchgedrungen. Auch die Europäer werden somit von ihren Rastern beeinflusst und vor allem junge Menschen werden von ihr mehr oder weniger subtil angesprochen, bestimmte Weltanschauungen und Handlungsmuster zu übernehmen. In diesem Zusammenhang ist der Roman Blackout des Österreichers Marc Elsberg meines Erachtens deswegen so bemerkenswert, weil er dem oben skizzierten Muster der omnipräsenten Superheldenerzählungen nicht entspricht. Erstens unterscheidet sich die im Text dargestellte Katastrophenursache deutlich von den meisten Darstellungen der heutigen Produktionen, und zweitens ist auch ihr Ausgang völlig anders. Denn in Blackout ist nicht nur die dargestellte Gefahr realistisch und aus diesem Grund auch für unsere Zivilisation als sehr ernst zu betrachten. Durchaus illusionslos und nüchtern, will heißen wenig optimistisch, ist auch jenes für das Finale des geschilderten Unheils vorgesehene Szenario.

Im Folgenden werde ich Schritt für Schritt zeigen, welche Faktoren es sind, die dazu beitragen, dass sich dieser Text von den meisten heutigen Katastrophendarstellungen in der Literatur grundsätzlich unterscheidet und somit sehr weit von dem von Jewett und Lawrence kritisierten Modell entfernt ist. Darüber hinaus werde ich kurz auch die Hauptbotschaften einer solchen Erzählung ansprechen.

\footnotetext{
${ }^{9}$ Vgl. Burdziej 2005.
} 


\section{Blackout und der, Wissenschaftsdiskurs'}

Der Roman von Elsberg ist nicht ausschließlich als realistisch zu bezeichnen. Es ist nicht bloß die Sache der Ästhetik oder des Stils der Prosa. Dem Autor zufolge soll der Text ein durchaus reales Risiko zeigen. Deswegen stützt sich das Konzept des Buches auf eine tiefgründige wissenschaftliche Recherche des Schriftstellers ${ }^{10}$ und weist ausdrückliche Bezüge zur heutigen technologischen sowie wissenschaftlichen Entwicklung auf. Dadurch aber, dass der Text eines jener möglichen Modelle schildert, die sich auf eine konkrete und breit genutzte Technologie (intelligente Stromzähler) beziehen, und das zudem von Wissenschaftlern und Politikern wahrgenommen und mit großer Aufmerksamkeit rezipiert wird, lässt sich der Roman auch an und für sich als ein unbestreitbarer Beitrag zum gegenwärtigen Wissenschaftsdiskurs lesen. Insofern stellt er ein schlagendes Beispiel dafür dar, dass Literatur per se imstande ist, spezifisches Wissen zu generieren. ${ }^{11}$

So ist die im Roman dargelegte Geschichte zwar fiktiv, aber die dargestellte Technologie ist absolut real. In technologischer Hinsicht wäre ein ähnlicher Cyberangriff auch in unserer Welt durchaus denk- und machbar. Im Nachwort betont der Autor:

Blackout ist Fiktion. Doch während meiner Arbeit am dem Manuskript wurde meine Fantasie mehrmals von der Realität eingeholt. So sah mein erster Entwurf 2009 eine Manipulation der SCADA-Systeme von Kraftwerken vor. Zu diesem Zeitpunkt hielten selbst Fachkreise diese Möglichkeit für kaum umsetzbar oder gänzlich abwegig - bis 2010 Stuxnet entdeckt wurde. Ähnlich war es mit der Gefahr, die von den Notkühlsystemen der Kernkraftwerke ausgeht - bis zur Katastrophe in Fukushima. (Elsberg 2012: 798)

Kurz vor Fertigstellung des Manuskripts des Buches im Mai 2011 wurden die Recherchen des Autors, was er selbst im Nachwort erwähnt (Elsberg 2012: 798), durch einen Bericht von Experten des Ausschusses für Bildung, Forschung und Technikfolgenabschätzung Gefährdung und Verletzbarkeit moderner Gesellschaften - am Beispiel eines großräumiges und lang andauernden Ausfalls der Stromversorgung bestätigt (Petermann 2010). Besonders die von Elsberg vorgesehenen und literarisch bearbeiteten Auswirkungen eines großflächigen und langfristigen Stromausfalls decken sich in überraschender Weise zu einem Großteil mit jenen, die von den Autoren des erwähnten Berichtes angegeben werden. Auch Jochen Homann, Präsident der Bundesnetzagentur ${ }^{12}$, gibt zu, dass ,,[d]ie Auswirkungen Herr Elsberg

10 Der Autor zählt im Nachwort auf: „Ich sprach mit Experten, etwa aus der Energie- und der IT-Branche sowie aus dem Katastrophenschutz.“ Und er fügt hinzu: „Sie gaben zwar alle bereitwillig Auskunft, namentlich genannt werden wollte niemand. Kein Wunder, bei den Informationen, die sie mir teils anvertrauten." (Elsberg 2012: 798)

11 Dies knüpft an eine breitere Diskussion über die Relation von Wissen und Literatur an. Siehe dazu z.B.: Borgards/Neumeyer (2013).

${ }^{12}$ Bundesnetzagentur (BNetzA) - Bundesnetzagentur für Elektrizität, Gas, Telekommunikation, Post und Eisenbahnen. 
gut recherchiert [hat]“ (Kutter/Rauner 2012), und sagt: „Ich habe das Buch zum Anlass genommen, um meinen Mitarbeitern Fragen zur Sicherheit unserer Stromnetze zu stellen. Dass Kraftwerke mit einer Software gesteuert werden, die angreifbar ist, hielt ich zunächst für reine Fiktion - da wurde ich eines Besseren belehrt" (Kutter/ Rauner 2012). Was sowohl der Bericht des Ausschusses für Bildung, Forschung und Technikfolgenabschätzung als auch der Roman von Elsberg besonders klar macht, ist vor allem die starke und unter normalen Umständen den Lebensstandard erhöhende Vernetzung der sog. Kritischen Infrastrukturen ${ }^{13}$ (wozu u.a. Informationstechnik und Telekommunikation, Transport und Verkehr, Energieversorgung oder das Gesundheitswesen zählen) der Grund dafür, dass die Auswirkungen eines Blackouts potenziell so dramatisch wären. Denn die Kritischen Infrastrukturen sind eben ,[...] aufgrund ihrer internen Komplexität sowie der großen Abhängigkeit voneinander hochgradig verletzbar" (Petermann 2010: 3).

Und so treten im Roman die Folgen des großräumigen Stromausfalls abrupt auf und sind massiv - und zwar beinahe alle Konsequenzen, die im Bericht beschrieben werden. Der Autor zeigt systematisch, wie ohne Strom nach und nach alle Lebensbereiche des heutigen Menschen zusammenbrechen, denn, was auch im Bericht hervorgehoben wird, ,[d]ie verschiedenen Sektoren Kritischer Infrastrukturen sind umfassend von einer kontinuierlichen Stromversorgung abhängig" (Petermann 2010: 5). Elsberg veranschaulicht auch die gesamteuropäische Vernetzung der Stromlinien (die heutzutage längst Realität geworden ist):

Strom schoss heute mehr denn je quer durch ganz Europa, von dort, wo er erzeugt wurde, dahin, wo gerade Bedarf herrschte. Konnten etwa die Österreicher mit ihren Wasserkraftwerken während der Spitzenlastzeiten am Abend nicht genug Elektrizität erzeugen, floss einfach die Energie slowakischer Atomkraftwerke in die Haushalte der Alpenrepublik. Ein paar Stunden später halfen kalorische Kraftwerke aus Spanien den Franzosen aus der abendlichen Belastung. Ein ständiges Geben und Nehmen. So verteilte sich die Elektrizität laufend gleichmäßig in ganz Europa, über die Hochspannungsnetze bis in die regionalen Verteilernetze, und wahrte das sensible Gleichgewicht zwischen Stromerzeugern und Konsumenten. (Elsberg 2012: 19)

Aber nicht nur die Auswirkungen eines andauernden und großflächigen Stromausfalls wurden im Roman treffend dargelegt. Auch fungiert der Cyberangriff seit langem als eine durchaus mögliche Ursache für den Blackout, was mehrmals im oben genannten Bericht betont wird. ${ }^{14}$ Im Gespräch mit Marc Elsberg

13 Die Autoren des genannten Berichts schreiben in diesem Zusammenhang von einem „Verletzlichkeitsparadox“: „Gerade weil in den meisten fortgeschrittenen Staaten die Stromversorgung relativ zuverlässig über lange Zeiträume funktioniert und nahezu alle technischen Systeme und sozialen Handlungen auf dieser relativen Verlässlichkeit aufbauen, steigt die Verletzbarkeit. Dieses ,Verletzlichkeitsparadox ' bedeutet, dass, wenn Versorgungsleistungen zunehmend weniger störanfällig organisiert werden, ,sich jede Störung von Produktion, Vertrieb und Konsum der Versorgungsleistungen umso stärker [auswirkt]'." (Petermann 2010: 32)

${ }^{14} \mathrm{Im}$ Bericht steht z.B.: „Als Ursachen für einen langandauernden und regional übergreifenden Stromausfall kommen u.a. technisches und menschliches Versagen, kriminelle oder terroristische Aktionen, Epidemien, Pandemien oder Extremwetterereignisse infrage." (Petermann 2010: 5) 
und Jochen Homann, dem Präsidenten der Bundesnetzagentur, wird Bezug auf die intelligenten Stromzähler genommen, die im Roman manipuliert werden und es den Hackern ermöglichen, den Blackout zu vollziehen. Die Interviewer Inge Kutter und Max Rauner bemerken, dass sogar das FBI gewarnt habe, ,[...] man könne diese Smart Meter von außen manipulieren“ (Kutter/Rauner 2012). Und obwohl Homann versichert, dass ,[f]ür vernetzte Smart Meter intensiv an Vorgaben zum Datenschutz und zur Datensicherheit gearbeitet [wird], sodass die Gefahr von Angriffen minimiert wird“, bleibt es für alle Gesprächspartner klar, dass ,[man] ganz sicher solche Stromzähler nie [wird] machen können“ (Kutter/Rauner 2012). ${ }^{15}$

Im Gegensatz zu den heutzutage dominierenden, von der amerikanischen Massenkultur beeinflussten Katastrophendarstellungen stellt Blackout von Elsberg somit ein Beispiel für jene Literatur dar, die nicht nur der Unterhaltung dient, sondern sowohl für die Wissenschaft von Relevanz ist als auch darauf abzielt, breitere Kreise von Lesern auf eine nicht rein fiktive, sondern reale Bedrohung aufmerksam zu machen. Hierfür werden im Roman - gleichsam wie in einem Gedankenlabor - die aktuellsten wissenschaftlichen Erkenntnisse literarisch verarbeitet und auf diese Weise gleichzeitig popularisiert. Gerade dieses macht das innovative Potential des Textes aus, weil er sich nicht nur als spannendes Unterhaltungsbuch liest, sondern sich ebenfalls in den Wissenschaftsdiskurs einschreibt.

\section{Das Figurenarsenal in Blackout}

Im Gegensatz zu den Superheldennarrationen, in denen die dargestellte Welt in der Regel schwarz-weiß ist und die Rollen der „Guten“ und „Bösen“ von vornherein klar verteilt sind (was oft schon anhand des Aussehens zum Ausdruck gebracht wird - die Guten sind in der Regel gutaussehend, die Bösen dagegen hässlich, was gewissermaßen die Gewalt den letzteren gegenüber rechtfertigt), ist die Frage nach der Verantwortlichkeit für die Katastrophe in Blackout viel komplexer. Das innovative Moment in Elsbergs Roman beruht maßgeblich auf der Komplexität der Stränge, und dadurch auf der Multiperspektivität und der Vielfalt der im Roman berücksichtigten Stimmen. Jeder der Protagonisten hat in der Geschichte seine mehr oder weniger überzeugende Sicht- und Argumentationsweise, aber auch Vorschläge, wie die bestehenden Probleme zu lösen sind. Diese enorme Perspektivenvielfalt korrespondiert dabei sehr präzise mit allen durchaus vorstellbaren Argumenten und Einstellungen, die bei einer derartigen Katastrophe und dem aus ihr resultierenden - wiederum absolut möglichen - Interessenkonflikt eintreten können. Auch dadurch grenzt sich der Text von Elsberg wieder deutlich von dem Schwarz-Weiß-Schema $\mathrm{ab}$, dass den amerikanischen Superheldengeschichten zugrunde liegt.

15 Siehe auch den Artikel von Joshua Pennell, einem Profi-Hacker, der schon 2010 Europa vor „smarten“ Stromnetzen warnte: Pennell (2010). 
Denn zum einen sind beispielsweise die Terroristen im Roman keine „Monster". Nicht nur sehen sie gut aus. Viel wichtiger ist, dass sie aus ideellen Gründen handeln. Alles, was sie tun, unternehmen sie nicht, um einfach das Böse zu tun oder weil sie eindeutig böse sind, was oft der Fall bei Superheldengeschichten ist, sondern um einer Idee willen. Sie handeln, wie einer der Initiatoren des Terroristenangriffs sagt, im Namen der

Menschen, die genug haben von der Art, wie die westliche Zivilisation und der Raubkapitalismus sie knechten und ausbeuten. Die es satthaben, beherrscht, belogen und ausgeraubt zu werden von einer kleinen Gruppe von Verbrechern, die sich Politiker, Banker und Manager betiteln. Die die feige Trägheit in den Reihensiedlungen und Wohnsilos und Bürofabriken nicht mehr ertragen. (Elsberg 2012: 780)

Einer der Protagonisten des Romans, der wesentlich zur Enttarnung der Terroristen und somit zur Bewältigung der Katastrophe beiträgt - ein Italiener namens Piero Manzano - ist ein Ex-Hacker, der sich vor Jahren an der Grenze der Legalität bewegte; gegen korrupte Politiker in Genua protestierend. Im gewissen Sinne stellte er sich also damals in eine Reihe mit den Menschen, die Jahre später für den Blackout verantwortlich zeichnen sollten. Sehr aussagekräftig sind daher die allerletzten Passagen des Romans. Da wird die Situation einige Tage nach der Wiederherstellung einer Grundversorgung mit Elektrizität kurz dargestellt. Als die Täter bekannt wurden, richtete sich der Zorn der Menschen zuerst nur gegen sie. Doch wenig später fangen die Menschen an, gegen die öffentlichen Stellen zu demonstrieren, ,[...] die im Voraus die Katastrophe nicht verhindert hatten und nun die gewohnten Verhältnisse längst nicht so schnell wiederherstellten, wie die Bevölkerung es erwartete" (Elsberg 2012: 796). Die Hauptprotagonisten beobachten die Proteste in Mailand und kommentieren diese untereinander folgendermaßen:

,Ich glaube nicht, dass dieser Pucao [der Hauptinitiator des Terroristenaktes - A.B.] recht hat", sagte sie [die schwedische Freundin von Piero Manzano - A.B.] und betrachtete die Demonstranten, klein wie Ameisen, auf dem Domplatz.

,Ich auch nicht. Wir können es anders, besser.“

Er [Piero Manzano - A.B.] ließ seinen Blick über das Panorama gleiten, legte seinen Arm um ihre Taille.

,Deshalb gehen wir jetzt wieder da hinunter auf die Straße zu den anderen.' (Elsberg 2012: 796)

Der Roman zeigt, dass Ziele und Ideen der Terroristen sowie jene derjenigen, die gegen sie kämpfen und die Katastrophe verhindern wollen, im Grunde genommen gleich - oder zumindest ähnlich - sind. Doch die von den Hackern gewählten Maßnahmen könne man nicht annehmen: friedliche Demonstrationen sowie Proteste seien angebracht und keineswegs ein rücksichtsloser Angriff auf ganze Gesellschaften. Dieser Gedanke steht auch in einer der polnischen Rezensionen des Romans im Vordergrund: „Das Interessanteste ist jedoch, dass der Autor - obwohl die Internet-Terroristen die Bösen sind, weil durch sie Hunderttausende ums Leben kommen - ausdrücklich andeutet, dass sie Recht haben. Wenn auch in der 
Frage der Ziele, und nicht der Maßnahmen“ (Łada 2015). ${ }^{16}$ Liest man zwischen den Zeilen, wird einsichtig, dass die Ursache der in Blackout dargestellten Katastrophe - was aber die Verantwortlichkeit der Terroristen nicht im Geringsten mindert - auch anderswo zu suchen sei: Die Regierenden, ihre Fehlentscheidungen, Schwächen und Missbräuche, aber auch unsere oft unbeholfen eingegangenen Kompromisse und Wahlen, all dies führe unmittelbar zu extremen Schritten der anderen Seite, die sich gegen die daraus resultierenden und für sie inakzeptablen Folgen verzweifelt zu wehren versucht. Jede menschliche Aktion erwecke immer eine Re-Aktion, eine Gegen-Aktion. Der Hauptgrund für die Katastrophe sei also der Mensch an sich, mit seinen menschlichen Eigenschaften, d.h. seinem menschlichen Dasein schlechthin: mit seinen besten Seiten, aber auch den bösen Charakterzügen und Reaktionen. Deswegen zeichnen sich die dargestellten Protagonisten durch alle möglichen Grautöne aus: Es gibt nur wenige, denen man - in moralischer Hinsicht - gar nichts vorwerfen könnte. Auf diese Weise wird beim Leser der Realitätseindruck verstärkt; unwillkürlich kommt ihm immer wieder der Gedanke in den Sinn: „So funktioniert es doch auch im Leben!“”

\section{Das Ende der Katastrophe}

Es gibt im Roman keinen Superhelden, der Europa und die USA vor der Katastrophe infolge des Blackouts von alleine retten könnte. Fast keine wichtige Entscheidung und Lösung erscheint hier als einfach und unkompliziert. Alle demokratisch gewählten Behörden und staatliche wie auch lokale Institutionen versuchen, die Folgen der Katastrophe zu tragen, was jedoch bald fast unmöglich ist, weil sich ihr Ausmaß als zu groß erweist. Die Kräfte aus ganz Europa und den Vereinigten Staaten sowie alle internationalen Organisationen (darunter Europol und Interpol) bündeln ihre Bemühungen und arbeiten in enger Kooperation, auch wenn es dabei zu unvermeidlichen Fehlern und manchmal auch zum Missbrauch des jeweiligen Amtes kommt. Wenn es also letztendlich zur Enttarnung der Terroristen und zur Wiederherstellung der Versorgung mit Elektrizität kommt, ist das der Mühe vieler demokratischer Institutionen zu verdanken, und keinem Superhelden mit übernatürlichen Fähigkeiten. Für den verständlichen Bedarf der Belletristik gibt es zwar im Roman einige Figuren, die sich im Plot als mehr oder weniger entscheidend erweisen (wie z.B. Piero Manzano, der als Ex-Hacker bei der Entschlüsselung der Cyberterroristen wesentlich hilft). ${ }^{17}$ Aber der Text gibt deutlich zu verstehen, dass sich bei der Bewältigung der Katastrophe die staatlichen, demokratischen Kräfte als unersetzlich erweisen. So wird auch angedeutet - was im Hinblick auf die wissenschaftlichen Voraussetzungen und Vermutungen bezüglich einer solchen

16 Übersetzung aus dem Polnischen: A. Burdziej.

${ }^{17}$ Für den bloßen Bedarf der Belletristik enthält der Text auch eine Liebesaffäre zwischen Protagonisten. 
bisher hypothetischen Situation durchaus begründet ist - dass die Folgen eines derartigen Stromausfalls über Jahrzehnte spürbar bleiben:

Trotz einer Grundversorgung mit Elektrizität war die allgemeine Versorgungslage in vielen Regionen noch immer schlecht, die Unfälle in den Kernkraftwerken und Chemiefabriken hatten ganze Landstriche unbewohnbar gemacht und Millionen aus ihrer Heimat vertrieben. Die Wirtschaft war auf Jahre ruiniert, eine gewaltige Depression wurde erwartet. Noch immer gab es keine endgültigen Todeszahlen, die Rede war von Millionen, wenn man Europa und die USA zusammenzählte, Langzeitopfer nicht eingerechnet. (Elsberg 2012: 796)

\section{Schlusswort: Blackout als „Warnvision“?}

Derzeit kann man ein wachsendes Interesse an Superheldennarrationen sowie allerlei absurden Katastrophengeschichten beobachten. Vermutlich sollte man es als Effekt der wachsenden Terrorismusgefahr und des damit verbundenen Gefühls der Bedrohung betrachten. Denn die Sehnsucht nach dem Sicherheitsgefühl lässt in den westlichen Gesellschaften das Bedürfnis nach einem manichäischen Weltbild gedeihen: Angesichts der sich wiederholenden terroristischen Anschläge kommen in den Menschen extreme Gefühle zum Ausdruck - und gleichzeitig eine mehr oder weniger bewusste Sehnsucht nach starken Persönlichkeiten, „wahren“ Helden, unerschrockenen Rettern und mächtigen „Führern“. Das wiederum geht mit wachsendem Populismus einher, der schnelle Lösungen propagiert, prämiert und keinen Raum für Verhandlungen, Dialog und Kompromiss zulässt, so dass bis zu einem gewissen Grad auch ,prophylaktische“ Gewalt (in breitem Sinne) gerechtfertigt wird.

Aus diesem Grund und in dieser Hinsicht sind Szenarien wie Blackout von Elsberg besonders bemerkenswert. Vor dem Hintergrund der Superhelden und des Überangebotes an Geschichten, in denen Katastrophen und ihr Ausgang unrealistisch sowie karikaturistisch dargestellt werden, helfen solche Entwürfe Vertrauen in den demokratischen Staat und seine Institutionen aufzubauen. Denn sie veranschaulichen die gegenwärtigen Bedrohungen realistisch, konkret und illusionslos, obgleich es durchaus möglich ist, dass ein derartiges Szenario nie eintritt. Auch der Autor hebt dies mit folgenden Worten hervor: „Das dargestellte Szenario ist eines von den vielen möglichen, denn niemand kann voraussehen, was sich in solch einer Situation ereignen würde" (Elsberg 2012: 798).

Der Roman Blackout von Elsberg ${ }^{18}$ lässt sich daher als gewisse „Warnvision“ betrachten. Sie basiert grundsätzlich auf einer tiefgründigen wissenschaftlichen Recherche und ist in großem Maße dank des allgemeinen Zugangs zu Wissen und Information möglich. Momentan bewegt sich dieses Genre in Richtung Wissenschaftsthriller. Aber auch der erkenntnisorientierten hard Science Fiction ist sie

${ }^{18}$ Und auch seine späteren Romane Zero (2014) und Helix (2016). 
nicht ganz fremd. ${ }^{19}$ Denn nicht jedes SF-Werk spielt in der fernen Zukunft oder handelt von dieser (Steinmüller 1995: 11). Erkenntnisorientierte SF betrachtet das SF-Werk vor allem als geistiges Labor für Gedankenexperimente, in dem der Autor die Funktion des Experimentators übernimmt (Steinmüller 2010: 20). Von Herbert W. Franke zum Beispiel wird die SF als „eine belletristische Ausprägung der Modellmethode: Technikfolgenabschätzung unter Berücksichtigung sozialer und emotionaler Aspekte“ interpretiert (Steinmüller 1995: 57). Genau so ist es bei Elsberg, dessen erzählte Geschichte auf einer Extrapolation bereits vorhandener technischer Entwicklungen beruht, wobei eine Vergrößerung der Maßstäbe vorgenommen wird. So wird in diesem Zusammenhang von mehreren Autoren die Bedeutung der SF als eines „Frühwarnsystems der Menschheit“ betont. ${ }^{20}$ Dieser Aspekt ist auch im Fall des Romans von Elsberg relevant. Blackout will nämlich laut seinem Autor zum Nachdenken und zur aktiven Mitgestaltung der Zukunft mobilisieren, aber auch den notwendigen Diskurs zu mehreren wichtigen Themen anregen, weil trotz des Gefahren- und Katastrophenpotenzials ein diesbezügliches gesellschaftliches Risikobewusstsein erst in Ansätzen vorhanden ist. ${ }^{21}$ Als Beispiel dafür kann Elsbergs eigenes Engagement dienen. Der Österreicher ist inzwischen zum Gesprächspartner in Politik und Wirtschaft avanciert (vor allem zu den Themen Energie und Energiewende, IT-Sicherheit, Cyberterrorismus, Gentechnik und moderne Gesellschaften) und mit seinen Bestsellerbüchern (Blackout, Zero, Helix $^{22}$ ) wurde der Autor zum Vermittler zwischen der Welt der Wissenschaft und Politik einerseits und der eines technologischen Laien andererseits.

Aber auch wichtige soziale und gesellschaftliche Botschaften sind in Elsbergs Romanen angelegt. Blackout ist außerdem insofern von Bedeutung, als hier der Autor mehrere aufschlussreiche Bemerkungen eingeflochten hat - unter anderem einen Gedanken, der in einer der polnischen Rezensionen erwähnt wird:

[...], dass manchmal der Idealismus von Bomben mörderischer ist, und von der Tyrannei ihn fast nichts mehr unterscheidet. [...]. Dass Staaten nicht dazu existieren, um einfach Staaten zu sein, sondern dazu, damit die Menschen ungefährdet leben können - der Mangel an dem Sicherheitsgefühl der Menschen bedeutet, unabhängig von der Staatsform, das Staatsende. (Lada $2015)^{23}$

In der wissenschaftlichen Darlegung argumentiert man oft mit schwer verständlichen Begriffen oder einer erdrückenden Zahl von abstrakten Daten. Laut Herbert W. Franke, einem österreichischen Wissenschaftler, Sachbuchautor und Science-Fiction-Schriftsteller fehle es vielen Menschen an Vorstellungskraft, um

${ }^{19}$ Hard SF als Subgenre der SF stützt sich inhaltlich und rhetorisch auf die Wissenschaft. Vgl. z.B. Steinmüller (1995: 34).

${ }^{20}$ Vgl. z.B. Hauss (2010); Steinmüller (1995); Lisek (2008).

${ }^{21}$ Was die Autoren des in diesem Aufsatz mehrmals zitierten Berichts zum Ausdruck bringen. Vgl. Petermann (2010: 7).

${ }^{22}$ Elsberg (2012); Elsberg (2014); Elsberg (2016).

23 Übersetzung aus dem Polnischen: A. Burdziej. 
die Formeln und Daten der (Zukunfts-)wissenschaft zu konkretisieren. Aber das gerade könne mithilfe der Dramatisierung der SF-Literatur gelingen, betont er (Franke 1976). ${ }^{24}$ Und dies gehört auch zur Mission, die Marc Elsberg vor seinem (SF-?) Roman stellen will. Seine letzten Worte im Nachwort zur Taschenbuchausgabe von Blackout lauten wie folgt: „Sollte Blackout neben spannenden Stunden auch etwas Wissen oder sogar einen kleinen Denkanstoß gegeben haben, freue ich mich darüber besonders“ (Elsberg 2012: 800). Zwar spielt also Elsbergs Prosa mit zeittypischen gesellschaftlichen Ängsten - und mit der gegenwärtigen „Lust an der Angst. “25 Gleichwohl thematisiert sie auch die heikelsten Fragen der technologischen Entwicklung. Und das ist wiederum kein Spiel mehr, sondern ein konkreter Beitrag, um die Menschen für die Risiken der neuesten Technologien zu sensibilisieren.

\section{Literatur}

\section{Quellen}

Elsberg, Marc (2012): Blackout-Morgen ist es zu spät. München.

\section{Sekundärliteratur}

Alewyn, Richard (1974): Die Lust an der Angst. In: Alewyn, Richard: Probleme und Gestalten. Essays. Frankfurt am Main. S. 307-330.

Bendyk, Edwin (2016): W poszukiwaniu utraconej przyszłości. In: Magia utopii. W poszukiwaniu utraconej przyszłości, Niezbędnik inteligenta. Sonderbeilage zur Wochenzeitschrift Polityka 2, S. 15.

Borgards, Roland / Neumeyer, Harald (2013): Literatur und Wissen. Ein interdisziplinäres Handbuch. Stuttgart/Weimar.

Burdziej, Stanisław (2005): Rezension zu: Jewett, Robert / Lawrence, John Shelton (2004), Captain America and the Crusade Against Evil: The Dilemma of Zealous Nationalism. Grand Rapids/ London. In: Paedagogia Christiana 1(15), S. 256-259.

Elsberg, Marc (2014): Zero - Sie wissen, was du tust. München.

Elsberg, Marc (2016): Helix - Sie werden uns ersetzen. München.

Franke, Herbert W. (1976): Science-fiction - Grenzen und Möglichkeiten. In: Jb. d. Arbeitskreises f. Jugendliteratur 3, S. 121-127.

Jewett, Robert / Lawrence, John Shelton (1997): The American Monomyth. New York.

Jewett, Robert / Lawrence, John Shelton (2004): Captain America and the Crusade Against Evil: The Dilemma of Zealous Nationalism. Grand Rapids/London.

\footnotetext{
${ }^{24}$ Zit. nach: Steinmüller (1995: 56 f.).

25 Zur Kategorie „Lust an der Angst“” vgl. u.a. Alewyn (1974).
} 
Kutter, Inge / Rauner, Max (2012): „Das wäre ein Riesenproblem“. Ein Gespräch mit Jochen Homann und Marc Elsberg. In: Zeit Online 6.12.2012, http://www.zeit.de/2012/50/Stromversorgung-Winter-Blackout-Marc-Elsberg-Jochen-Homann/komplettansicht?print (Zugriff am 8.01.2018).

Lawrence, John Shelton / Jewett, Robert (2002): The Myth of the American Superhero. Grand Rapids.

Lisek, Marcin (2008): Science fiction - zludzenie czy proroctwo przyszłości? In: Środkowoeuropejskie Studia Polityczne (1), http://ssp.amu.edu.pl/archive/nr-12008 (Zugriff am 8.01.2018).

Łada, Wojciech (2015): BLACKOUT. Ktoś zgasit światto, https://wpolityce.pl/kultura/253565-blackout-ktos-zgasil-swiatlorecenzja (Zugriff am 8.01.2018).

Pennell, Joshua (2010): Smart Meter: Dann schalten Hacker die Lichter aus. In: Zeit Online 29.04.2010, http://www.zeit.de/digital/internet/2010-04/smartgrid-strom-hacker/komplettansicht (Zugriff am 8.01.2018).

Petermann,Thomas et al. (2010): Gefährdung und Verletzbarkeit moderner Gesellschaften - am Beispiel eines großräumigen Ausfalls der Stromversorgung. TAB-Arbeitsbericht Nr. 141. Berlin.

Sembritzki, Katja (2012): „Blackout“ legt Europa lahm. Erst fällt der Strom aus, und dann? In: n-tv Nachrichtenfernsehen GmbH 28.05.2012, https://www.n-tv.de/leute/buecher/Erst-faellt-derStrom-aus-und-dann-article6249461.html (Zugriff am 8.01.2018).

Steinmüller, Karlheinz (1995): Gestaltbare Zukünfte. Zukunftsforschung und Science Fiction. Gelsenkirchen.

Steinmüller, Karlheinz (2010): Science Fiction: eine Quelle von Leitbildern für Innovationsprozesse und ein Impulsgeber für Foresight. In: Hauss, Kalle et al.: Foresight - Between Science and Fiction. IFQ-Working Paper No. 7. Bonn. S. 19-31.

Walter, Francois (2010): Katastrophen. Eine Kulturgeschichte vom 16. bis ins 21. Jahrhundert. Stuttgart.

Zwierzchowski, Marcin (2016): Śnimy już tylko koszmary. In: Magia utopii. W poszukiwaniu utraconej przyszłości, Niezbędnik inteligenta. Sonderbeilage zur Wochenzeitschrift Polityka 2, S. 100-104. 